\title{
Improved antimycobacterial activity of rifampin using solid lipid nanoparticles
}

\author{
Ehsan Aboutaleb ${ }^{1}$, Massoumeh Noori', Narges Gandomi', Fatemeh Atyabi ${ }^{2}$, Mohammad Reza Fazeli ${ }^{3}$, \\ Hossein Jamalifar ${ }^{3}$ and Rassoul Dinarvand ${ }^{1,2^{*}}$
}

\begin{abstract}
Rifampin (RIF) is one of the front-line drugs in therapy of tuberculosis (TB). The emergence of multidrug-resistant strains of mycobacteria has greatly contributed to the increased incidence of TB. Nano-based formulation of several antimicrobials has been shown to improve either antibacterial efficacy or pharmacokinetic behavior. In this study, RIF-loaded solid lipid nanoparticles (SLNs) were prepared by a modified microemulsion-based method and their particle size, zeta potential, encapsulation efficiency, morphology, and antibacterial activity against Mycobacterium fortuitum were evaluated. The resulting SLNs were spherical with diameter of about $100 \mathrm{~nm}$, with low negative zeta potential, and an encapsulation efficiency of $82 \%$. The formulation also sustained the drug release for $72 \mathrm{~h}$. The antimycobacterial efficacy was greatly improved against $M$. fortuitum, and the minimum inhibitory concentration of drug-loaded SLNs was eight times less than free RIF. Drug-free SLNs and the ingredients showed no antibacterial effect. It can be concluded that as expected, solid lipid nanoparticles are promising vehicles for enhanced antimycobacterial effect of rifampin.
\end{abstract}

Keywords: Solid lipid nanoparticles, Rifampin, Antibacterial activity, Mycobacteria, Mycobacterium fortuitum, MIC, Drug delivery, Tuberculosis

\section{Background}

Although tuberculosis (TB) is as old as humanity, it is still one of the most widespread and fatal diseases [1]. One-third of the world's total population (more than two billion) are infected with mycobacterium tuberculosis [2]. Although it is more common in under developed and developing countries, it also remains one of the major concerns among developed countries as well, due to growing incidences of AIDS [3]. It is reported that in 2007, about 1.77 million people have died from $\mathrm{TB}$, and it is estimated that about 9.27 million new TB cases occur annually [3]. Complete eradication of the disease in most cases is not successful due to multidrugresistant strains of TB. Also, patients may terminate the treatment because of side effects, prolongation of therapy, or relief of the symptoms [4].

\footnotetext{
* Correspondence: dinarvand@tums.ac.ir

${ }^{1}$ Novel Drug Delivery Systems Laboratory, Faculty of Pharmacy, Tehran

University of Medical Sciences, Tehran 1417614411, Iran

${ }^{2}$ Nanotechnology Research Centre, Faculty of Pharmacy, Tehran University of Medical Sciences, Tehran 1417614411, Iran

Full list of author information is available at the end of the article
}

The nanoparticulate drug delivery systems can improve the quality of antibacterial treatment by decreasing side effects and reducing the frequency of dosing via prolongation of drug residence time [5]. Solid lipid nanoparticles (SLNs) are nano-sized drug delivery vectors which hold the advantages of lipid emulsions, liposomes, and polymeric nanoparticles while avoiding numerous disadvantages of these carriers. SLNs are made from physiological lipids, so there is a very low concern about their safety and biocompatibility [6]. Additionally, they can carry both lipophilic and hydrophilic drugs [7] and possess a solid matrix to control the release rate of loaded drug [8]. They can be produced on large scale and sterilized by different procedures with conventional equipment that are used for the production of lipid-based infusions [9]. Therefore, SLNs are considered as suitable drug carriers for intravenous administration [10].

Rifampin (RIF) is one of the first-line drugs in therapy of TB. It is a bactericidal antibiotic, which binds to DNA-dependent RNA polymerase and inhibits the initiation of RNA synthesis process. It is a wide spectrum antibiotic but is used most commonly in the treatment 
of tuberculosis [11]. Resistance to this antibiotic is rising in mycobacterium species, and this phenomenon has been received with great concerns about the therapy of $\mathrm{TB}$ in the future [3]. Many multiple drug-resistant mycobacteria have emerged, and their incidents are constantly increasing [3]. Two mechanisms are considered to be involved in the natural drug resistance of mycobacteria: the mycobacterial cell wall permeability barrier and the active multidrug efflux pumps [12].

The preparation of several nano-sized formulations of RIF has been reported before, and most of them were successful in either increasing the antimycobacterial efficacy of RIF or improving its pharmacokinetics [13-17]. Pandey et al. encapsulated three frontline antituberculosis drugs (rifampin, isoniazid, and pyrazinamide) in poly (lactic-co-glycolic acid nanoparticles and used it orally against Mycobacterium tuberculosis infection in mice. Compared to the solution of the same drugs with same dosage, the outcome of the study was the improvement of pharmacokinetics, and the authors concluded that these polymeric nanoparticles could replace 28 conventional doses with only three doses [18]. This group also successfully utilized the same oral antituberculosis drugs incorporated in solid lipid nanoparticles against mice tuberculosis, and the formulation was also able to reduce dosing frequency [19]. However, this study was not able to discriminate between the effects of three individual drugs, and only the combination effect was studied. Although better pharmacokinetic profile was contributed to the better performance of the formulation, no direct antimicrobial challenge tests were provided to examine the direct antimicrobial performance on Mycobacteria.

Several studies have revealed the ability of SLNs to deceive P-glycoprotein (P-gp) pump and reduce efflux by this molecule [20]. P-gp efflux is one of the mechanisms of multidrug-resistance not only at the level of $M$. tuberculosis but also at the level of somatic cells [21]. Additionally, Bargoni et al. showed that tobramycinloaded SLNs concentrate in lungs and concluded that this increased concentration might help in treating pulmonary infections [20].

Cerebral tuberculosis infection is a fatal situation that requires rapid medical intervention; one of the drugs used in this case is IV RIF; however, the drug cannot pass the blood-brain barrier (BBB) completely and the therapy is troublesome [22]. It has been shown before that SLNs can pass the BBB efficiently [23]. Thus, RIFloaded SLNs can also be useful for brain-targeted drug delivery in case of cerebral tuberculosis.

TB infection in some cases can spread from lungs to the lymphatic system. So, drug delivery to macrophages is of crucial importance in TB therapy. SLNs can aid the delivery of RIF to the lungs as well as to the lymphatic system [24]. When the SLNs enter the lungs, alveolar macrophages phagocyte them and transfer them to the lymphoid tissues [25].

Several studies have shown that the body distribution of the drug is highly influenced by the size and surface characters of the SLNs [26]. The nanoparticles with size of about $100 \mathrm{~nm}$ and coated with PEG or PEG-like surfactants (Tween 80, Poloxamer 188, etc.) can escape the RES system and remain in the blood circulation for a longer time [27].

The goal of this study was to produce RIF-loaded SLN formulation suitable for intravenous administration. Therefore, efforts were made to achieve a formulation with the ideal size of about $100 \mathrm{~nm}$ and below and the highest encapsulation efficiency. A modified microemulsion method was utilized to produce high-concentration SLN formulation.

\section{Methods}

\section{Materials}

RIF was a gift from Hakim Pharmaceutical Co. (Tehran, Iran). Cetyl palmitate and Tween 80 were purchased from Merck KGaA (Darmstadt, Germany). Poloxamer 188 was obtained from Synopharm GmbH (Barsbuttel, Germany). Lowenstein-Jensen medium was from Merck, Amicon ${ }^{\circledR}$ Ultra-15 centrifuge tubes were from Millipore Corporation (MA, USA). M. fortuitum (ATCC 2701P) was provided by the stock culture of Department of Drug and Food Control, Tehran University of Medical Sciences (Tehran, Iran). Freshly prepared reverse osmosis (RO) water was used in all experiments. All other chemicals were either reagent or analysis grade.

\section{Preparation of SLNs}

SLNs were prepared by a modified microemulsion-based technique. Generally, microemulsion methods are based on dilution of a warm microemulsion of drug agent, lipid, surfactant, and water in a bulk of cold water and the resulting formulation usually contains a large extent of water which is difficult to separate. By adding an extra ultrasonic treatment, the amount of diluting cold water could be decreased, while the acceptable particle characteristics were saved.

The lipid (cetyl palmitate) and the emulsifiers (Tween $80 /$ Poloxamer 188) were molten and mixed by means of magnetic stirring. RIF was added to this mixture and suitably stirred at $80^{\circ} \mathrm{C}$ to ensure that the drug is totally dispersed; then, $25 \mathrm{ml}$ of $\mathrm{RO}$ water at the same temperature was added. This coarse pre-emulsion was treated with ultrasonic (13 watts $/ 10 \mathrm{~min} / 80^{\circ} \mathrm{C}$ ) using the ultrasonic probe sonication system (Misonix Inc., NY, USA); the resulting microemulsion was immediately dispersed in cold deionized water while stirring $\left(2^{\circ} \mathrm{C}\right.$ to $\left.3^{\circ} \mathrm{C}\right)$. 


\section{Size, zeta potential, morphology, and stability determinations}

The mean aerodynamic diameter (MAD), and polydispersity index (PDI) were determined with the aid of photon correlation spectroscopy (Zetasizer Nano ZS, Malvern Instruments, Worcestershire, UK) at $25^{\circ} \mathrm{C}$. Zeta potential was also determined by the above mentioned device. Before the measurements, samples were diluted appropriately with $\mathrm{RO}$ water to prevent interparticle scattering which can interfere the results of measurements. Each sample was analyzed three times and mean $\pm \mathrm{SD}$ is reported.

Semicontact (tapping) mode atomic force microscopy (AFM) (Dualscope/Rasterscope C26, Danish Micro Engineering, Copenhagen, Denmark) with rectangular cantilever (length $230 \mu \mathrm{m}$, width $40 \mu \mathrm{m}$, thickness $7 \mu \mathrm{m}$ ), conical tip (height 15 to $20 \mu \mathrm{m}$, angle $<20^{\circ}$, curvature radius $<10 \mathrm{~nm}$ ), and resonance frequency of 150 to 190 $\mathrm{kHz}$ was used to investigate the morphology and size of the nanoparticles. Vacuum-dried samples mounted on a glass cover slip were used for imaging, and areas of $5 \times$ $5 \mu \mathrm{m}$ were scanned with resolution of less than $0.1 \mathrm{~nm}$.

To investigate the stability of the formulations, the samples of each formulation were filled into glass vials and stored in either controlled room temperature or in a refrigerator $\left(4^{\circ} \mathrm{C}\right)$. After fixed time intervals $(4,24,72$, and $148 \mathrm{~h}$ ), the samples were inspected visually for any possible cake forming, and size measurements were carried out as mentioned above. Formulations with no cake forming, and size increase of less than $10 \%$ during the $148 \mathrm{~h}, 72 \mathrm{~h}, 24 \mathrm{~h}$ were regarded as stable.

\section{Drug content and encapsulation efficiency measurement}

The encapsulation efficiency was indirectly determined by measuring the concentration of unloaded drug in the dispersion medium. Freshly prepared SLN dispersions $(2 \mathrm{ml})$ were placed in Amicon ${ }^{\circledR}$ Ultra-15 tubes. Using an ultracentrifuge (Sigma 3k30, Sigma Laboratory, Osterode, Germany) at $6,000 \mathrm{~g}$ for $15 \mathrm{~min}$, the nanoparticles were separated from the medium. The filtrate was then diluted with $\mathrm{RO}$ water (if necessary), and the RIF concentration was measured using visible spectrophotometry (CE7500, Cecil Instruments, Cambridge, UK) using a calibration curve of rifampin in deionized water at $336 \mathrm{~nm}$. The encapsulation efficiency was calculated using the below equation.

$$
\left(\begin{array}{c}
\text { Total amount of drug used for the preparation of } \\
\text { SLNS }- \text { amount of unloaded drug }
\end{array}\right) / / 100
$$

\section{In vitro drug release}

In vitro drug release studies were performed by a dialysis bag method using a 12,000-dalton molecular weight cut- off membrane to retain the nanoparticles, and phosphate buffer saline (PBS) $\left(\mathrm{pH} 7.4,37^{\circ} \mathrm{C}\right)$ as the release medium. SLNs dispersion $(10 \mathrm{ml})$ was placed in the dialysis bag (Sigma Laboratories, Osterode, Germany), and both ends of the bag were clamped. The bag was soaked in $200 \mathrm{ml}$ release medium. A thermostatic shaker (Unimax 1010, Heidolph Instruments $\mathrm{GmbH} \&$ Co. KG, Schwabach, Germany) at $37^{\circ} \mathrm{C}$ and rate of 36 cycles per min was used to mimic biological condition. At fixed time intervals $(0,0.5,1,2,4,6,8,24,48,72$, and $120 \mathrm{~h}), 5 \mathrm{ml}$ portions of the medium were removed, and fresh medium was added to maintain sink condition. Released drug concentration was determined by spectrophotometry at $336 \mathrm{~nm}$ using a standard curve of RIF in PBS. All the operations were carried out in triplicate.

\section{DSC investigations}

A Mettler DSC 823 (Mettler Toledo International, Inc., D-Gießen, Germany) was utilized to investigate the dispersion of the drug in the nanoparticles and the degree of crystallinity of lipid core. Degree of crystallinity was evaluated using the following formula by Freitas and Muller [28]:

$$
\mathrm{CI} \%=100 \times \frac{\Delta \mathrm{H}_{\mathrm{SLN}} \text { aqueous dispersion }}{\left(\Delta \mathrm{H}_{\text {bulk }} \times \text { lipid concentration }\right)}
$$

The SLNs were freeze-dried prior to analysis using a Christ alpha 2-4 LD freeze drier (Martin Christ GmbH, Osterode, Germany). Precisely weighted freeze-dried SLNs (approximately $10 \mathrm{mg}$ ) and each of the ingredients were filled separately in 40- $\mu \mathrm{l}$ aluminum pans; using appropriate equipment, the pans were sealed and then heated in comparison to an empty pan as a reference from $30^{\circ} \mathrm{C}$ to $310^{\circ} \mathrm{C}(10 \mathrm{~K} / \mathrm{min})$ under dry nitrogen purge $(80 \mathrm{ml} / \mathrm{min})$.

\section{Antibacterial activity study}

Well diffusion method was utilized to investigate the antimicrobial efficacy of the RIF-SLNs. Aqueous solution of RIF with concentration equal to SLNs and drug-free SLN (made exactly the same as RIF-SLNs without adding the drug) were used as control groups. Using $M$. fortuitum (ATCC 2701P) as a closely related mycobacterium to $M$. tuberculosis, the antibacterial efficacy was investigated.

The bacterial suspension with the turbidity comparable to 0.5 McFarland was transferred onto the surface of Lowenstein-Jensen plate using sterile cotton swab. RIF-loaded SLNs and aqueous solutions of RIF with concentration of $220 \mu \mathrm{g} / \mathrm{ml}$ were prepared, then serial dilutions were made from the stock. Diameter wells $(8 \mathrm{~mm})$ were bored on the medium with a cork borer, and 100 $\mu \mathrm{l}$ aliquots of each diluted sample were introduced into each well. As control group, $100 \mu \mathrm{l}$ of drug-free SLNs 
were delivered into 3 wells. After 48 -h incubation at $37^{\circ} \mathrm{C}$, the growth of bacteria around the wells was evaluated, and the minimum inhibitory concentrations (MICs) of RIF-SLNs and RIF aqueous solution were determined as the lowest concentration that could inhibit bacterial growth around the well.

\section{Results}

Size, zeta potential, morphology, and stability results The size (MAD) and zeta potential of some selected formulations are presented in Table 1. Employing more than $1.5 \mathrm{~g}$ cetyl palmitate in the formulation led to increased sizes and unstable dispersions. At least $2 \mathrm{~g}$ Tween 80 was necessary to stabilize the formulation, but increasing its amount had no more effect (data not shown). Adding Poloxamer 188 to the formulation led to slight increase of the size, but up to $10 \%$ of total surfactant, the effect was negligible. The addition of Poloxamer 188 contributed to more stable formulations; also, zeta potential of Tween 80-based nanoparticles were slightly negative $(-7 \mathrm{mV})$, and by increasing the percent of Poloxamer 188, the zeta potential became more negative $(-12 \mathrm{mV})$.

Dilution ratios of 1:2 and less showed acceptable quality, but below this ratio (e.g., 1:1) showed either poor stability or increased sizes. So, the formulation with $1.5 \mathrm{~g}$ cetyl palmitate, 2 g Tween 80, 0.2 g Poloxamer 188, and dilution ratio of 1:2 was selected for further investigations. It is also noteworthy that without the ultrasonic treatment, dilution ratios of down to 1:10 demonstrated poor characteristics (data not shown).

Stability of almost all dispersions in room temperature was poor, but in $4^{\circ} \mathrm{C}$, the stability was acceptable for most of the formulations especially those which contained Poloxamer 188 (Table 1). Adding the drug (80 mg rifampin $\mathrm{HCl}$ ) to the preparation process increased the size by 3 to $17 \mathrm{~nm}$, but no other change in stability or zeta potential of the SLNs was demonstrated; this result has been reported by other authors as well $[29,30]$. Figure 1 shows the AFM topography images of

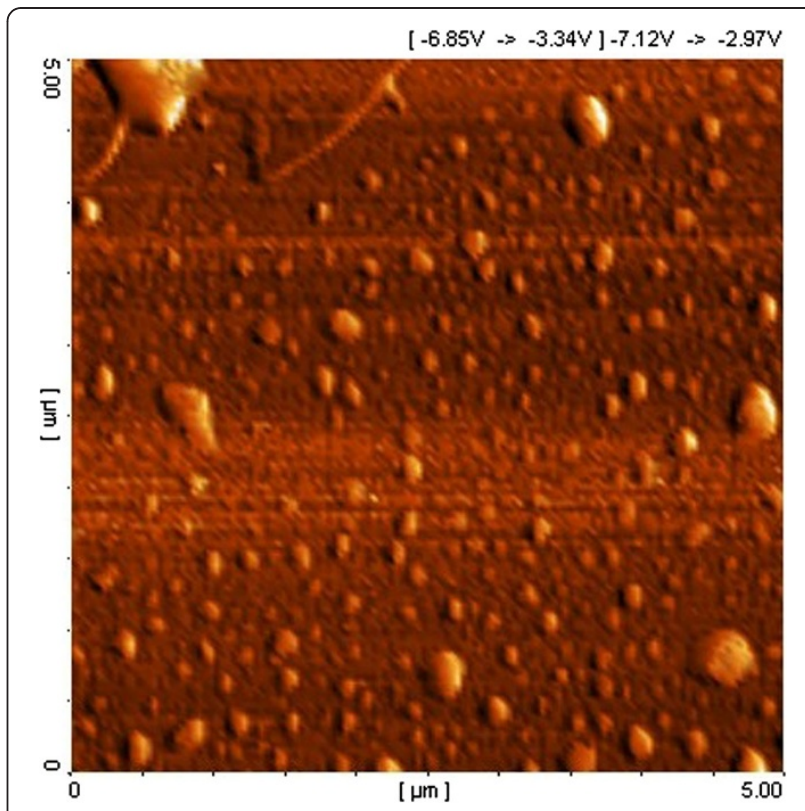

Figure 1 AFM image of rifampin-loaded SLN formulation (F8).

SLNs. It clearly shows the spherical nanoparticles with almost matching dimensions of PCS data.

\section{Drug content and encapsulation efficiency results}

Due to the high solubility of RIF in the lipid core, the encapsulation efficiency was good enough for all the formulations. For the selected formulation, an encapsulation efficiency of approximately $82 \%$ was achieved. Drug loading also reached up to $5 \% w / w$.

\section{In vitro release study}

The cumulative release profile of the selected formulation is shown in Figure 2. Release of RIF from SLNs was evaluated over a $120-\mathrm{h}$ time period. The profile showed a biphasic pattern; a burst release of about $13 \%$ of the drug in the first hour is believed to be related to unloaded drug and the drug molecules that are adsorbed

Table 1 Physicochemical characters of some rifampin-loaded formulations

\begin{tabular}{|c|c|c|c|c|c|c|c|c|}
\hline & Cetyl palmitate (g) & $\mathrm{T} 80(\mathrm{~g})$ & Poloxamer 188 (g) & Dispersion ratio & Mean diameter $(\mathrm{nm}) \pm \mathrm{SD}$ & PDI & Zeta potential & Stability $^{a}$ \\
\hline F1 & 1 & 2 & & $1: 2$ & $102 \pm 4$ & 0.2 & $-8.34 \pm 1$ & $*$ \\
\hline $\mathrm{F} 2$ & 1 & 2 & & $1: 1$ & $311 \pm 20$ & 0.33 & $-7.17 \pm 4$ & - \\
\hline F3 & 1 & 3 & & $1: 4$ & $89 \pm 4$ & 0.16 & $-11 \pm 2$ & $* *$ \\
\hline F4 & 1 & 4 & & $1: 4$ & $101 \pm 4$ & 0.2 & $-12.1 \pm 1$ & $* *$ \\
\hline F5 & 1 & 4 & & $1: 8$ & $104 \pm 3$ & 0.3 & $-13.7 \pm 0.5$ & $* *$ \\
\hline F6 & 1.5 & 2 & & $1: 2$ & $87.8 \pm 3$ & 0.3 & $-11 \pm 0.4$ & $* *$ \\
\hline F7 & 1.5 & 4 & 0.2 & $1: 2$ & $99.3 \pm 4.0$ & 0.18 & $-10.3 \pm 0.5$ & $* * *$ \\
\hline F8 & 1.5 & 2 & 0.2 & $1: 2$ & $108.7 \pm 5.5$ & 0.18 & $-10.7 \pm 0.5$ & $* * *$ \\
\hline
\end{tabular}

a $24 \mathrm{~h}\left({ }^{*}\right), 72 \mathrm{~h}\left({ }^{* *}\right), 148 \mathrm{~h}\left({ }^{* *}\right)$. 


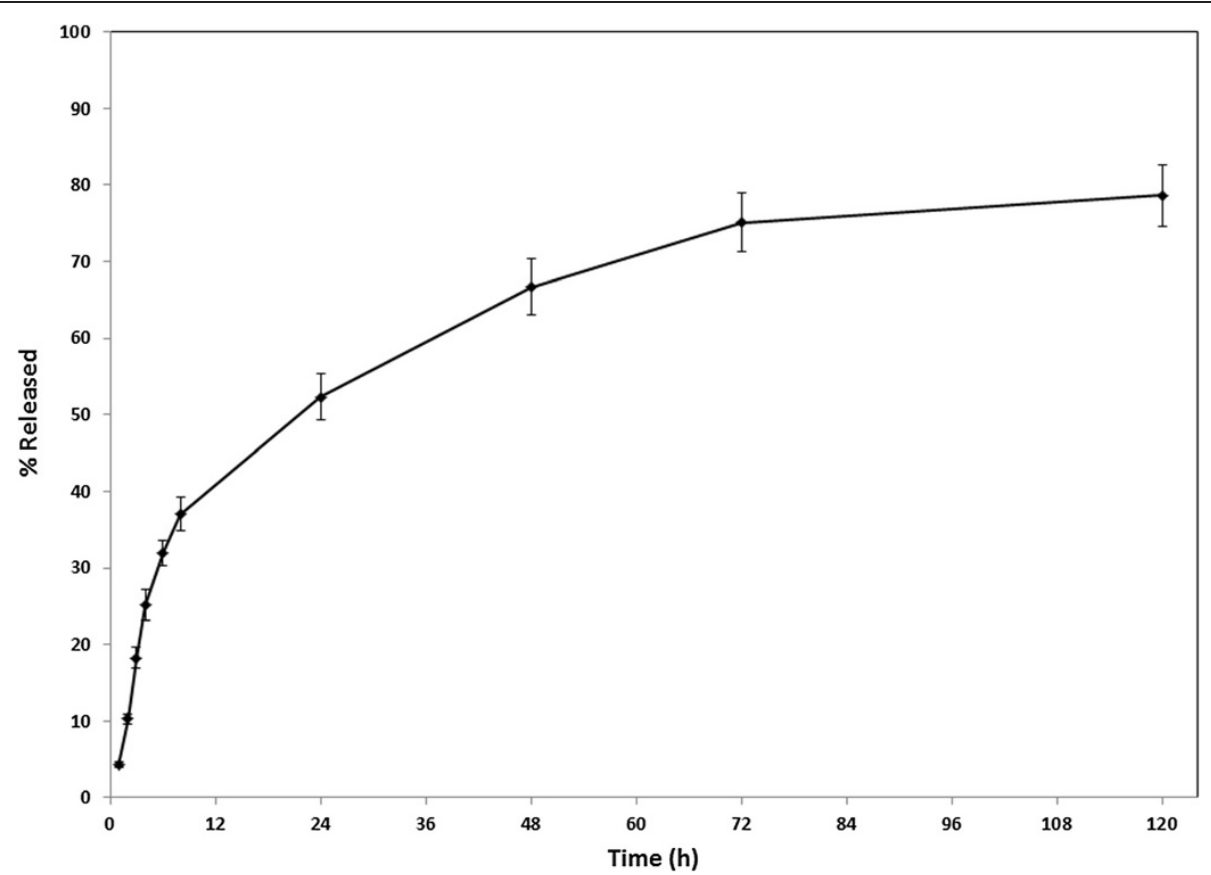

Figure 2 In vitro drug release from rifampin-loaded SLN formulation (F8) in PBS ( $\mathrm{pH}, 7.4)$.

to the SLNs surface, and then a slow release of the loaded drug. The release profile reaches the plateau (about $75 \%$ of total drug) in about $72 \mathrm{~h}$.

\section{DSC investigation results}

Thermograms of freeze-dried RIF-SLN, cetyl palmitate, Tween 80, and RIF are shown in Figure 3. Cetyl palmitate has a melting point of about $60^{\circ} \mathrm{C}$, and it is clearly noticeable in SLNs and cetyl palmitate bulk thermograms. RIF has characteristic endothermic and exothermic peaks from $190^{\circ} \mathrm{C}$ to $260^{\circ} \mathrm{C}$, but the peaks are not visible in the RIF-SLN thermogram. This indicates that the drug is not in crystalline form and is molecularly dispersed in the nanoparticle matrix. Crystallinity index was calculated based on the previously mentioned formulae, and it was found that the lipid core of the nanoparticles was $43 \%$ crystalline.

\section{Antibacterial activity results}

Regarding the high encapsulation efficiency (almost $82 \%)$, the SLN dispersion, not freeze dried, was used for antibacterial efficacy test. A great improvement in the antimycobacterial activity of RIF was shown in this study. The RIF solution needed at least $22-\mu \mathrm{g} / \mathrm{ml}$ concentration to effectively inhibit the growth of the bacteria, while the SLN formulation could effectively inhibit the bacterial growth by the concentration of as low as $2.75 \mu \mathrm{g} / \mathrm{ml}$. This shows at least an eight-time efficacy improvement in comparison to RIF solution.
Drug-free SLNs were also prepared similar to the preparation of RIF-SLN formulation except no drug was used. This formulation had no effect on the bacterial growth, so the improved antibacterial efficacy is related to the ability of SLN formulation to deliver the drug efficiently to the bacteria and not to the antibacterial effect of any other ingredients (lipid or surfactants) in the formulation.

\section{Discussion}

Microemulsion-based methods have been utilized in several studies to produce SLNs [31-33]. In most of the microemulsion methods, warm microemulsion is dispersed in high amount of cold water (typical ratio 1:50), so the final formulation is much diluted. The removal process of excess water from the prepared SLN dispersion is a difficult task. In this study, a simple modification in the method for the preparation of SLNs helped to increase this ratio to $1: 2$, and utilizing ultrasonication to produce microemulsions could efficiently compensate the effect of high concentration on stability and size of the formulation. The resultant formulation was as stable as the traditional microemulsion formulations, and the size and zeta potentials were also acceptable.

In vitro studies revealed retarded drug release from the SLNs. About 13\% of drug was released in the first hour. This effect can be related to the adsorbed drug molecules on the SLNs surface and the unbound drug. By the end of $24 \mathrm{~h}$, about $50 \%$ of drug was released and 


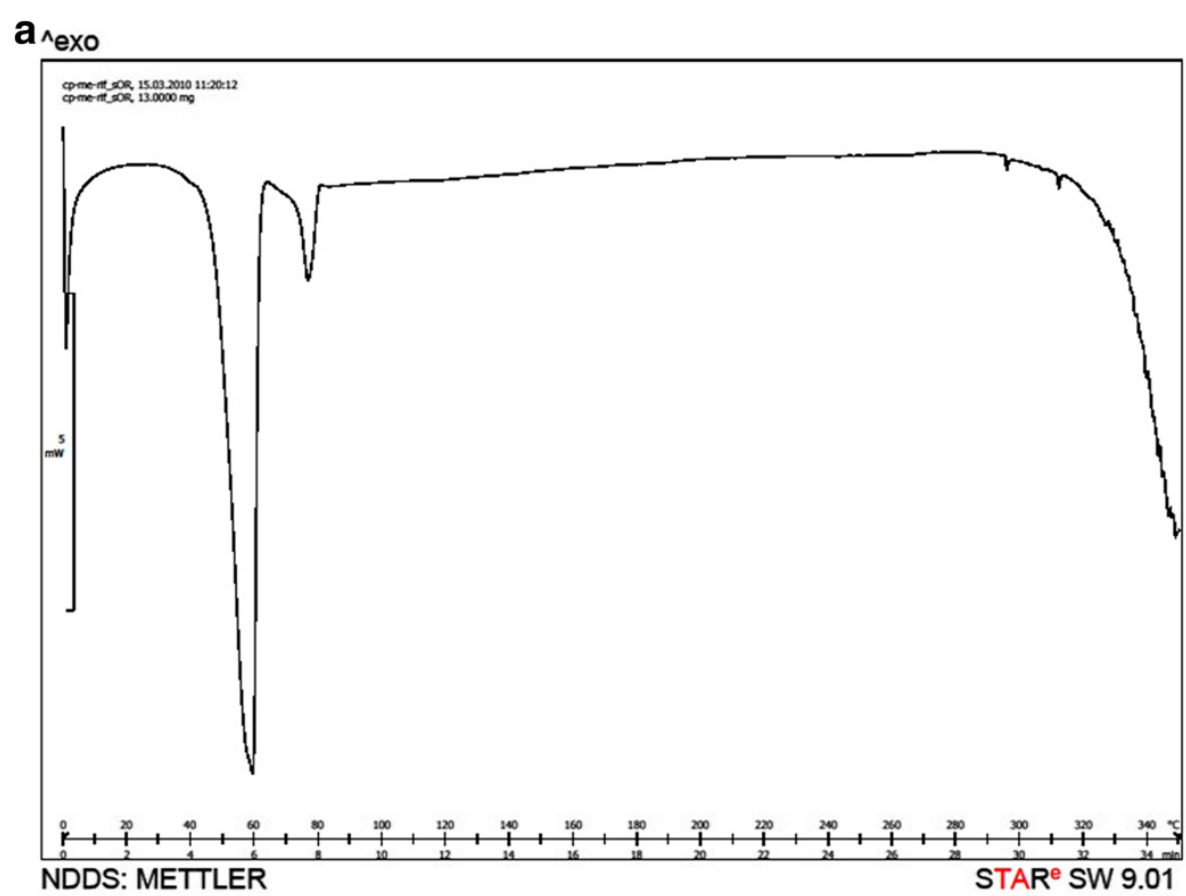

b

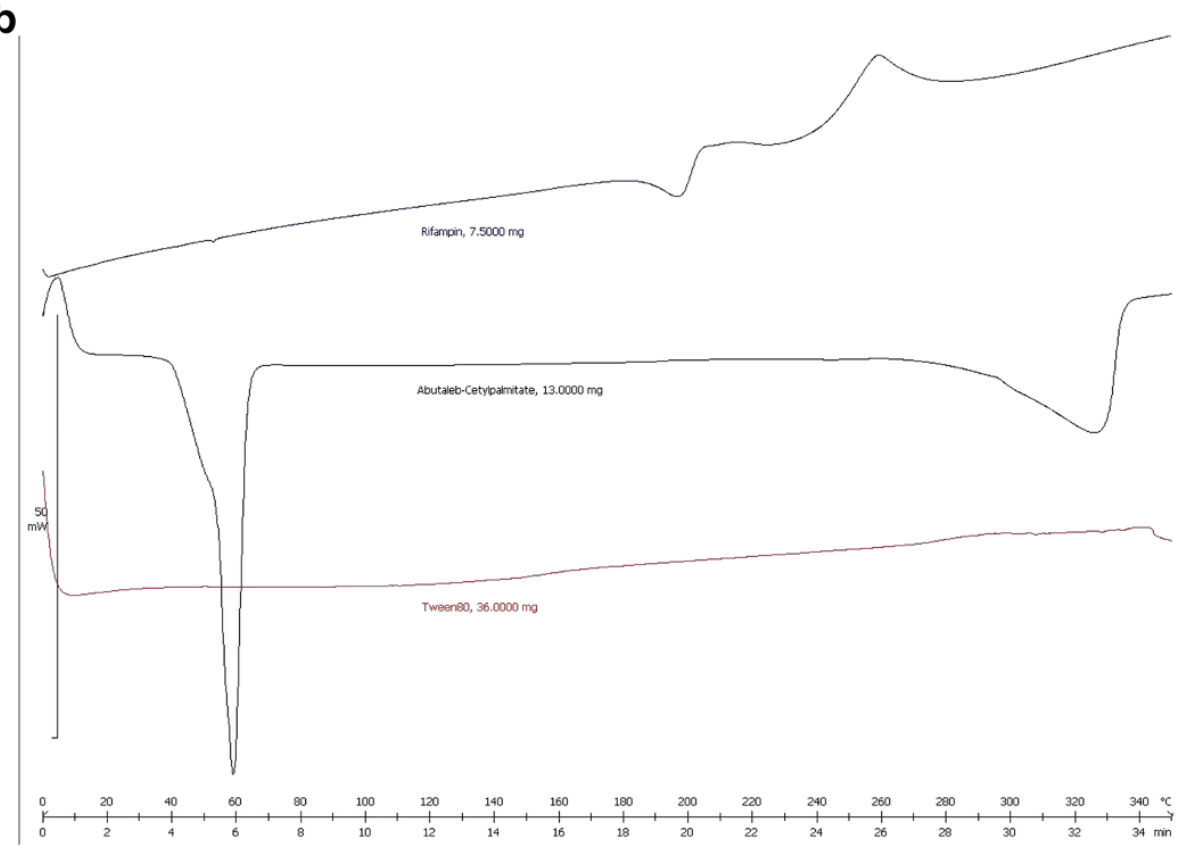

Figure 3 DSC thermograms of selected (a) SLN formulation (F8) and (b) bulk materials.

after about $72 \mathrm{~h}$ drug release reached a steady state (75\%). This indicates that this formulation can sustain the drug release in blood stream and hypothetically is able to lower dosing frequency.

The final formulation included rifampin $\mathrm{HCl}(80 \mathrm{mg})$, cetyl palmitate $(1.5 \mathrm{~g})$, Tween 80 ( $2 \mathrm{~g})$, and Poloxamer 188 (0.2 g). High lipid solubility of RIF led to high encapsulation efficiency for all the formulations (about $82 \%$ ), so there was no need to freeze dry SLNs for antibacterial activity tests; the tests were carried out on SLN dispersions. Results revealed that loading of RIF in SLNs improved MIC at least eight times compared to RIF solution against $M$. fortuitum. As mentioned above, two mechanisms are responsible for the drug resistance of mycobacteria: the mycobacterial cell wall permeability barrier and the active multidrug efflux pumps. SLN formulation can hypothetically manipulate both of them to increase the efficacy. 
RIF-SLNs may better penetrate the bacterial cell wall due to their small size and hydrophobic nature which is similar to gram negative bacterial cell wall. Also, SLNs can effectively hinder the effect of P-gp pumps [21]. Moreover, this formulation possibly will be able to decrease the administration frequency due to prolonged drug release.

\section{Conclusion}

SLN formulation of rifampin with high encapsulation efficiency was prepared by a microemulsion-based method with a satisfactory particle size range and drug release profile. The formulation consisted of cetyl palmitate $(1.5 \mathrm{~g})$ as the lipid core, Tween 80 $(2 \mathrm{~g})$, and Poloxamer $188(0.2 \mathrm{~g})$ as the surfactant. RIF-loaded SLNs were eight times more effective than RIF solution against M. fortuitum. This SLNbased formulation seems to be a good choice for antimycobacterial formulations.

\section{Competing interest}

The authors declare that they have no competing interests.

\section{Authors' contributions}

EA participated in the SLN preparation and characterization, and drafted the manuscript. MN carried out the experiments. NG participated in the SLN preparation and characterizations. FA was the co-supervisor of the project and reviewed the manuscript. MRF supervised the antimicrobial studies. HJ participated in the antimicrobial studies. RD conceived the study and participated in its design and coordination, and is the corresponding author of the manuscript. All authors read and approved the final manuscript.

\section{Authors' information}

EA is a Ph.D. candidate; MN and NG are both Pharm D students. FA is a professor of pharmaceutical nanotechnology. MRF is a professor of pharmaceutical microbiology. $\mathrm{HJ}$ is a research assistant to MRF. RD is professor of pharmaceutics and the dean of the Faculty of Pharmacy, Tehran University of Medical Sciences.

\section{Acknowledgments}

The authors are grateful for the financial support of Tehran University of Medical Sciences.

\section{Author details}

${ }^{1}$ Novel Drug Delivery Systems Laboratory, Faculty of Pharmacy, Tehran University of Medical Sciences, Tehran 1417614411, Iran. ${ }^{2}$ Nanotechnology Research Centre, Faculty of Pharmacy, Tehran University of Medical Sciences, Tehran 1417614411, Iran. ${ }^{3}$ Department of Drug and Food Control and Pharmaceutical Quality Assurance Research Center, Faculty of Pharmacy, Tehran University of Medical Sciences, Tehran 1417614411, Iran.

\section{Received: 25 October 2011 Accepted: 8 October 2012}

Published: 29 October 2012

\section{References}

1. Horsley, S, Barrow, S, Gent, N, Astbury, J: Informal care and psychiatric morbidity. J Public Health 20(2), 180-185 (1998)

2. Suarez, S, O'Hara, P, Kazantseva, M, Newcomer, CE, Hopfer, R, McMurray, DN, Hickey, AJ: Respirable PLGA microspheres containing rifampicin for the treatment of tuberculosis: screening in an infectious disease model. Pharm Res 18(9), 1315-1319 (2001)

3. WHO: Tuberculosis Facts. World Health Organization, Geneva (2009)

4. Pearson, ML, Jereb, JA, Frieden, TR, Crawford, JT, Davis, BJ, Dooley, SW, Jarvis, WR: Nosocomial transmission of multidrug-resistant Mycobacterium tuberculosis. A risk to patients and health care workers. Ann Intern Med 117(3), 191-196 (1992)
5. Zhang, L, Pornpattananangkul, D, Hu, C-M, J, Huang, C-M: Development of nanoparticles for antimicrobial drug delivery. Curr Med Chem 17(6), 585-594 (2010)

6. Mehnert, W, Mäder, K: Solid lipid nanoparticles: production, characterization and applications. Adv Drug Deliv Rev 47(2-3), 165-196 (2001)

7. Müller, RH, Mäder, K, Gohla, S: Solid lipid nanoparticles (SLN) for controlled drug delivery - a review of the state of the art. Eur J Pharm Biopharm 50(1), 161-177 (2000)

8. Müller, RH, Mehnert, W, Lucks, JS, Schwarz, C, zur Mühlen, A, Meyhers, H, Freitas, C, Rühl, D: Solid lipid nanoparticles (SLN)—an alternative colloidal carrier system for controlled drug delivery. Eur J Pharm Sci 41, $62-69$ (1995)

9. Schwarz, C, Mehnert, W, Lucks, JS, Müller, RH: Solid lipid nanoparticles (SLN) for controlled drug delivery. I. Production, characterization and sterilization. J Control Release 30(1), 83-96 (1994)

10. Wissing, SA, Kayser, O, Müller, RH: Solid lipid nanoparticles for parenteral drug delivery. Adv Drug Deliv Rev 56(9), 1257-1272 (2004)

11. Seth, S: Textbook of Pharmacology. Elsevier, India (2008)

12. De Rossi, E, Aínsa, JA, Riccardi, G: Role of mycobacterial efflux transporters in drug resistance: an unresolved question. FEMS Microbiol Rev 30(1), 36-52 (2006)

13. Briones, E, Colino, Cl, Lanao, JM: Delivery systems to increase the selectivity of antibiotics in phagocytic cells. J Control Release 125(3), 210-227 (2008)

14. Durán, N, Alvarenga, MA, Da Silva, EC, Melo, PS, Marcato, PD: Microencapsulation of antibiotic rifampicin in poly(3-hydroxybutyrate-co-3hydroxyvalerate). Arch Pharm Res 31(11), 1509-1516 (2008)

15. Esmaeili, F, Hosseini-Nasr, M, Rad-Malekshahi, M, Samadi, N, Atyabi, F Dinarvand, R: Preparation and antibacterial activity evaluation of rifampicinloaded poly lactide-co-glycolide nanoparticles. Nanomed Nanotech Biol Med 3(2), 161-167 (2007)

16. Saraogi, GK, Gupta, P, Gupta, UD, Jain, NK, Agrawal, GP: Gelatin nanocarriers as potential vectors for effective management of tuberculosis. Int J Pharm 385(1-2), 143-149 (2010)

17. Skidan, IN, Gel'perina, SE, Severin, SE, Guliaev, AE: Enhanced activity of rifampicin loaded with polybutyl cyanoacrylate nanoparticles in relation to intracellularly localized bacteria. Antibiotiki I Khimioterapiya 48(1), 23-26 (2003)

18. Pandey, R, Zahoor, A, Sharma, S, Khuller, GK: Nanoparticle encapsulated antitubercular drugs as a potential oral drug delivery system against murine tuberculosis. Tuberculosis (Edinb.) 83(6), 373-378 (2003)

19. Pandey, R, Sharma, S, Khuller, GK: Oral solid lipid nanoparticle-based antitubercular chemotherapy. Tuberculosis (Edinb.) 85(5-6), 415-420 (2005)

20. Bargoni, A, Cavalli, R, Zara, GP, Fundaro, A, Caputo, O, Gasco, MR: Transmucosal transport of tobramycin incorporated in solid lipid nanoparticles (SLN) after duodenal administration to rats. Part II-tissue distribution. Pharmacol Res 43(5), 497-502 (2001)

21. Erokhina, MV, Aleksandrova, EA: In vitro development of rifampicin resistance in the epithelial cells. Problemy Tuberkuleza I Bolezner Legkikh 8, 58-61 (2006)

22. Harrison, AC: Treatment of tuberculosis. In: Ministry of Health of New Zealand (ed.) Guidelines for Tuberculosis Control in New Zealand 2003, chap. 16, pp. 1-36. Ministry of Health, Wellington (2003)

23. Kaur, IP, Bhandari, R, Bhandari, S, Kakkar, V: Potential of solid lipid nanoparticles in brain targeting. J Control Release 127(2), 97-109 (2008)

24. Pandey, R, Khuller, GK: Solid lipid particle-based inhalable sustained drug delivery system against experimental tuberculosis. Tuberculosis (Edinb.) 85(4), 227-234 (2005)

25. Gelperina, S, Kisich, K, Iseman, MD, Heifets, L: The potential advantages of nanoparticle drug delivery systems in chemotherapy of tuberculosis. Am J Respir Crit Care Med 172(12), 1487-1490 (2005)

26. Üner, M, Yener, G: Importance of solid lipid nanoparticles (SLN) in various administration routes and future perspectives. Int J Nanomed 2(3), 289-300 (2007)

27. Maldiney, T, Richard, C, Seguin, J, Wattier, N, Bessodes, M, Scherman, D: Effect of core diameter, surface coating, and PEG chain length on the biodistribution of persistent luminescence nanoparticles in mice. ACS Nano 5(2), 854-862 (2011)

28. Freitas, $\mathrm{C}$, Müller, RH: Correlation between long-term stability of solid lipid nanoparticles (SLN) and crystallinity of the lipid phase. Eur J Pharm Biopharm 47(2), 125-132 (1999) 
29. Lim, SJ, Kim, CK: Formulation parameters determining the physicochemical characteristics of solid lipid nanoparticles loaded with all-trans retinoic acid. Int J Pharm 243(1-2), 135-146 (2002)

30. Ugazio, E, Cavalli, R, Gasco, MR: Incorporation of cyclosporin A in solid lipid nanoparticles (SLN). Int J Pharm 241(2), 341-344 (2002)

31. Cavalli, R, Marengo, E, Rodriguez, L, Gasco, MR: Effects of some experimental factors on the production process of solid lipid nanoparticles. Eur J Pharm Biopharm 43, 110-115 (1996)

32. Gasco, MR: Solid lipid nanospheres from warm microemulsions. Pharm Technol Eur 9(11), 52-58 (1997)

33. Cavalli, R, Caputo, O, Marengo, E, Pattarino, F, Gasco, MR: The effect of the components of microemulsions on both size and crystalline structure of solid lipid nanoparticles (SLN) containing a series of model molecules. Pharmazie 53, 392-396 (1998)

doi:10.1186/2228-5326-2-33

Cite this article as: Aboutaleb et al.: Improved antimycobacterial activity of rifampin using solid lipid nanoparticles. International Nano Letters 2012 2:33.

Submit your manuscript to a SpringerOpen ${ }^{\circ}$ journal and benefit from:

- Convenient online submission

- Rigorous peer review

- Immediate publication on acceptance

- Open access: articles freely available online

- High visibility within the field

- Retaining the copyright to your article 